\title{
Intrahepatic cholestasis of pregnancy
}

\author{
Ana Maria SCUTELNICU ${ }^{1}$, Brindusa Ana CIMPOCA-RAPTIS ${ }^{1}$, Anca Marina CIOBANU ${ }^{1,2}$, \\ Nicolae GICA ${ }^{1,2}$, Radu BOTEZATU1 ${ }^{1,2}$, Corina GICA ${ }^{1}$, Gheorghe PELTECU1,2, Anca Maria PANAITESCU ${ }^{1,2}$ \\ ${ }^{1}$ Filantropia Clinical Hospital, Bucharest, Romania \\ 2"Carol Davila” University of Medicine and Pharmacy, Bucharest, Romania
}

\begin{abstract}
Intrahepatic cholestasis of pregnancy is the most common liver disease during pregnancy. The main features are maternal pruritus, in the absence of a rash, and raised serum bile acids. Intrahepatic cholestasis of pregnancy is associated with an increased risk of adverse perinatal outcomes, including preterm birth, meconium-stained amniotic fluid, fetal asphyxia with neonatal unit admission or even stillbirth. The main treatment option is ursodeoxycholic acid. We review the etiology, diagnosis, adverse pregnancy outcomes, focusing especially on management of this intriguing condition.
\end{abstract}

Keywords: cholestasis, pregnancy outcome, stillbirth, bile acids, pruritus

\section{INTRODUCTION}

Intrahepatic cholestasis of pregnancy (ICP) is the most common liver disease in pregnancy. It is a reversible event that usually resolves in 48 hours after delivery, but rarely it can take up to 6 weeks post-partum. ICP typically presents after 30 weeks, but it can present as early as 7 weeks of gestation.

The incidence of ICP increases with parity, multiple pregnancies, fertility treatments, maternal age (> 35 years) and family history of biliary disease. ICP rates differ globally being $0.7 \%$ in the UK, with a maximum general incidence reported in Chile of $1.5-4 \%$, while the Araucanos Indians in Chile have the highest incidence worldwide at 27.6 percent. The prevalence in European countries is about $1 \%$ [1].

ICP affects the liver with impaired bile flow, leading to elevated serum bile acids levels. Bile salts play an important role in lipids absorption and cell signaling as part of key metabolic process [2]. Cholestasis is characterized by an impairment of bile secretion and transport, leading to subsequent accumulation of toxic bile components in the body. In a physiological pregnancy, bile acids are transported from the fetus to the mother, whereas in a pregnancy complicated by ICP, transplacental transport occurs in the opposite direction. Therefore, levels of bile acids are increased in both the mother and the fetus. Elevated levels of total bile acids (TBA) are associated with increasing oxidative stress and apoptosis, thus damaging liver cells and other tissues.

\section{MATERIALS AND METHODS}

A PubMed search was completed using the keywords and medical subject heading (MeSH) "intrahepatic cholestasis" and "pregnancy". The search included randomized controlled trials, meta-analyses, clinical trials, systematic reviews, clinical practice guidelines, review articles and resulted in 332 articles, from which only 26 papers were further reviewed and included in the final review.

\section{ETIOLOGY}

ICP has a complex etiology with endocrine, genetic and environmental components. Concentrations of es- 
trogens, progesterone and their metabolites increase during pregnancy to reach peak levels during the third trimester and subsequently fall after birth, coinciding with the natural history of ICP and thus supporting the endocrine etiopathogenesis. Genetic susceptibility to the disorder is supported by evidence of familial clustering, increased risk in first-degree relatives, increased risk in some ethnic groups and a high recurrence rate (60 to 70\%) [3]. Several genes have been linked to familial intrahepatic cholestasis such as ATP8B1, ABCB11, ABCB4 and NR1H4.

The first paper addressing a possible genetic etiology for IHC was published in 1999 and it explored the link between $A B C B 4$ variants and ICP. Jacquemin et al. investigated the relatives of a patient with progressive familial intrahepatic cholestasis (PFIC), who was homozygous for a highly deleterious variant in ABCB4 (c.1712delT) and had undergone liver transplantation during childhood [4]. Six females in the family had at least one episode of ICP and in the four cases that genetic data was available, they were all found to be heterozygous for the ABCB4 variant [4]. Subsequent analysis of 31 patients with PFIC secondary to $A B C B 4$ variants revealed three mothers with $I C P$, all of whom were heterozygous for ABCB4 variants (pArg652Gly, pArg957Ter, c.1744delT). In a family with multiple members with ABCB4 variant pArg788Trp and chronic liver disease, all nine female members who survived to adulthood suffered from ICP, including both homozygotes and heterozygotes. In five of them their first presentation of liver disease was with ICP. These findings suggest that there is a predisposition to ICP in patients with a family history of liver disease who are heterozygous for ABCB4 variants [5]. Additionally, variations in NR1H4 gene, also known as farnesoid $X$ receptor (FXR) may be implicated in ICP, possibly via downregulation of BSEP expression [6,7].

\section{CLINICAL AND PARACLINICAL FINDINGS}

ICP is characterized by intense pruritus and elevated serum bile acids that typically appears in the third trimester. Pruritus usually begins on the palms and soles, but it can extend to the whole body, with no skin rash except scratch marks. It is worse at night, causing insomnia, irritability and even depression and it usually precedes the rise in biliary acids by a few days to a few weeks. The itching is presumably caused by bile acids deposition in the skin. Jaundice, right upper quadrant pain, nausea, poor appetite or steatorrhea may also occur [3]. Sometimes, fatty stools due to absorption disorders, especially lipid malabsorption, can appear. Consequently, there can be a deficiency of fat-soluble vitamins, especially vitamin $\mathrm{K}$, that can lead to elongated prothrombin times and causing hemorrhages.
Hyperlipidemia is characteristic of some, but not all cholestatic diseases. Serum cholesterol is elevated in cholestasis because its metabolic degradation and excretion are impaired. Bile is the normal excretory pathway for cholesterol, and with reduced bile formation, cholesterol is retained [8].

The most sensitive biochemical marker used in the diagnostics of intrahepatic cholestasis of pregnancy is serum total bile acids. Usually in pregnant women bile acids levels are slightly and insignificantly elevated than in non-pregnant women. The cut-off point is defined as $10 \mu \mathrm{mol} / /$ by most laboratories. Common serum markers raised in ICP are bile acids (up to 10-100 fold), transaminases (moderately, but there can be a 10-25-fold increase in rare cases), alkaline phosphatase and gamma-glutamyl transpeptidase (modestly in $30 \%$ of cases). The last increase of the last marker is unusual in the most other forms of cholestatic liver disease in which gamma-glutamyl transpeptidase (GGT) levels parallel other cholestatic markers. Sometimes an increased concentration of bile acids may be the only biochemical abnormality [3].

\section{DIAGNOSIS}

The diagnosis of ICP is based on the clinical presentation of pruritus without rash, abnormal liver function tests (elevated serum bile acids and transaminases) and exclusion of other causes of itching and abnormal liver function.

Differential diagnosis includes pruritus of other etiologies, hepatitis, preeclampsia or HELLP syndrome, acute fatty liver disease [10].

\section{ADVERSE PREGNANCY OUTCOME}

The intense pruritus of ICP is very unpleasant for mother, but the biggest concern is related to the perinatal complications such as preterm birth, meconium-stained amniotic fluid, fetal asphyxia with neonatal unit admission or even stillbirth. There are many studies that found a correlation between ICP and adverse pregnancy outcomes, but the exact mechanisms are yet to be clarified.

Meconium staining of amniotic fluid is present in approximately $15 \%$ of term pregnancies and it is considered a sign of fetal distress. Bile acids may cause fetal distress with subsequent meconium passage, but they are also known to increase colonic motility $[11,12]$. The etiology of fetal death is still poorly understood and it is thought to occur suddenly, probably because of an arrhythmogenic event $[1,13]$. Considering that studies on in vivo fetal cardiac cells are difficult to realize, the data available is derived from in vitro studies on neonatal rat cardiomyocytes. Models of the fetal heart 
TABLE 1. Differential diagnosis (DDX) of pruritus with no liver disease - adapted from [9]

\begin{tabular}{|c|c|c|c|c|}
\hline DDX & Timing & Skin lesions & Laboratory findings & Complications \\
\hline \begin{tabular}{|l|} 
Pruritus \\
gravidarum
\end{tabular} & $\begin{array}{l}\text { Second/Third } \\
\text { trimester }\end{array}$ & None & None & None \\
\hline $\begin{array}{l}\text { Atopic eruption of } \\
\text { pregnancy }\end{array}$ & First trimester & $\begin{array}{l}\text { Dry, red rash }+/- \text { small blisters } \\
\text { Typically affects trunk and limb flexures }\end{array}$ & None & None \\
\hline $\begin{array}{l}\text { Polymorphic } \\
\text { eruption of } \\
\text { pregnancy }\end{array}$ & Third trimester & $\begin{array}{l}\text { Urticarial papules or plaques, vesicles and } \\
\text { target lesions } \\
\text { Typically affects lower abdominal striae with } \\
\text { umbilical-sparing }\end{array}$ & None & None \\
\hline $\begin{array}{l}\text { Pemphigoid } \\
\text { gestationis }\end{array}$ & $\begin{array}{l}\text { Second/Third } \\
\text { trimester }\end{array}$ & $\begin{array}{l}\text { Itchy rash that develops into large, tense } \\
\text { blisters }\end{array}$ & $\begin{array}{l}\text { Rare autoimmune } \\
\text { condition } \\
\text { characterized by } \\
\text { complement-fixing } \\
\text { immunoglobulin G } \\
\text { antibodies }\end{array}$ & $\begin{array}{l}\text { Rash associated with } \\
\text { increased risk of preterm } \\
\text { delivery and SGA } \\
\text { Recurs in subsequent } \\
\text { pregnancies and } \\
\text { with combined oral } \\
\text { contraceptive } \\
\end{array}$ \\
\hline $\begin{array}{l}\text { Prurigo of } \\
\text { pregnancy }\end{array}$ & Third trimester & $\begin{array}{l}\text { Groups of red-brown papules on the } \\
\text { abdomen and extensor surfaces of the limbs } \\
\text { Papules may persist postpartum }\end{array}$ & None & None \\
\hline $\begin{array}{l}\text { Pruritic folliculitis } \\
\text { of pregnancy }\end{array}$ & Third trimester & $\begin{array}{l}\text { Acneiform eruption on the shoulders, upper } \\
\text { back, thighs, and arms } \\
\text { Follicular papules and pustules, which } \\
\text { may be filled with pus, but culture is } \\
\text { typically sterile; rash usually improves with } \\
\text { advancing gestation }\end{array}$ & None & None \\
\hline
\end{tabular}

(using cells from rodents) have shown that high bile acids levels (especially taurocholic acid) cause arrythmias leading to stillbirth. A possible mechanism is an altered calcium ion dynamic in the cardiomyocyte and impaired function of gap junctions, blocking the normal conduction with subsequent loss of synchronous contraction in the myocardium resulting mainly in ventricular tachycardia or fibrillation [14]. Mothers with ICP have not been reported to develop arrythmias so that could imply that fetal cardiomyocytes are more susceptible to erroneous conduction than adult cells, but more date is needed to sustain this hypothesis. There is an ongoing clinical trial aiming to assess whether sever ICP (bile acids $>=40 \mu \mathrm{mol} / \mathrm{l}$ ) is associated with abnormal fetal heart rhythms and abnormal myometrial contractility (available at: https://clinicaltrials.gov/ ct2/show/NCT03519399). Also, a dose-dependent vasoconstrictive effect of bile acids (mainly cholic acid) has been shown on human placental chorionic veins, possibly explaining the fetal distress, asphyxia and death [15] by an abrupt reduction of oxygenated blood at the placental site.

One of the first relevant studies that addressed the issue of adverse pregnancy outcomes in ICP was a prospective cohort study from Sweden that analyzed 45,485 pregnancies who were screened for this condition. Pruritus was present in $2.1 \%$ of cases and ICP was diagnosed in $1.5 \%$ of cases. This study showed a $1-2 \%$ increased risk of spontaneous preterm delivery, asphyxia events and meconium-stained amniotic fluid with every $1 \mu \mathrm{mol} / \mathrm{l}$ increase in maternal serum bile ac- ids over the $40 \mu \mathrm{mol} / \mathrm{l}$ cut-off. So, this study defined a mild and a severe form of ICP with important implications in disease management [13].

In 2019, an important meta-analysis was published in Lancet, which showed a clear association between women with the most severe disease (bile acids $\geq 100$ $\mu \mathrm{mol} / \mathrm{l}$ ) and increased stillbirth risk (in singleton pregnancies) compared with those with milder disease (bile acids $<100 \mu \mathrm{mol} / \mathrm{l}$ ) and the background population [16]. This offered a new cut-off for the management of ICP with hopes to reduce the increasing number of iatrogenic preterm births. This study clearly demonstrated that the risk of stillbirth is proportional to the serum bile acids level, but the actual number of cases given the percentage of $0.13 \%$ stillbirths for mild disease, 0.28 stillbirths for moderate disease and $3.44 \%$ for severe disease does not necessarily support the indication for early delivery in all those cases of ICP.

A large population-based study from Finland comparing women with and without an ICP diagnosis in 1972-2000 found a significant risk of later hepatobiliary disease for women diagnosed with ICP, in particular gallstone-related morbidities and hepatitis $C$ infection [17]. Another population-based cohort study examined 11,388 Swedish women with ICP and linked them to future development of hepatobiliary diseases (cholangitis, gallstones disease, cirrhosis and chronic hepatitis) through their nationwide patient registry. An ICP diagnosis was associated with an increased risk of later hepatobiliary disease with an increment of $1 \%$ per year [18]. A 2015 study using the same database found in- 
creased risks of hepatobiliary cancer, cardiovascular disease and immune-mediated diseases including diabetes mellitus, thyroid disease, psoriasis, inflammatory polyarthropathies and Crohn's disease [19].

For a patient with history of ICP, oral contraceptives with estrogens should be recommended with caution and at the lowest possible dose, given the risk for developing cholestasis outside of pregnancy. The past consensus has been that ICP is a contraindication for oral contraceptives. However, recent data suggests that the new low-dose pills can be safely used as long as liver function is normal and patients are informed of the possible risks [8].

\section{DIAGNOSIS AND MANAGEMENT}

A lack of consensus even in the diagnostic criteria contributes to the differences in management of ICP. For example, RCOG defines ICP as obstetrical pruritus accompanied by otherwise unexplained elevation in liver function tests and/or bile acid concentrations, both of which resolve after delivery. The elevated bile acid concentration is not necessarily required for diagnosis in this case and it is explicitly stated that normal levels do not exclude the diagnosis. They also state that a confirmed diagnosis can only be made following resolution of symptoms and laboratory values postpartum. Giving that the diagnostic criteria are different and not universally accepted, the management of the disease will be very different between health care systems. South Australia Maternal \& Neonatal Community of Practice (SAMNCP) and Society for Maternal-Fetal Medicine (SMFM) are the only guidelines to divide ICP into mild (bile acid concentrations $10-40 \mu \mathrm{mol} / \mathrm{l}$ ) and severe $(>40 \mu \mathrm{mol} / \mathrm{l})$ disease $[20,21]$. In our opinion, it is a very important classification in guiding clinician with further management.

Fasting bile acid measurements are sometimes performed and used to diagnose ICP, but random dosing is now generally used, being more convenient for both patient and practitioner. The differences between fasting and random bile acids levels are small and they do not support the change in recommendation [21]. Also, we are looking for the peak of bile acid level, which gives the risk for complications, so prandial bile acid measurement should be more specific. Cholic and chenodeoxycholic acid are the principal bile acids elevated in maternal serum of untreated patients and ursodeoxicholic acid (UCDA) constitutes $60 \%$ of bile acids after treatment is initiated. Changes in the total bile acid measurement reflect similar alterations in the concentrations of the pathologically elevated bile acids, e.g., a two-fold increase in enzymatic total bile acids is accompanied by approximately a two-fold increase in cholic acid and chenodeoxycholic acid at most UDCA doses, so enzymatic total bile acid assays are good predictors of both cholic acid and chenodeoxycholic acid [22].

The main treatment option recommended by most guidelines is oral ursodeoxycholic acid (UDCA), in doses varying from $1,000 \mathrm{mg}$ up to $2,500 \mathrm{mg} /$ day. It has been shown to improve cholestasis in primary biliary cholangitis and other hepatobiliary disorders since 1970.

The most important study to date, a multicenter, randomized placebo-controlled trial, that analyzed the effects of UDCA on perinatal complications (stillbirth, preterm delivery, neonatal unit admission) and serum bile acids concentration, transaminases and the maternal inch-score post-treatment, showed that UDCA does not reduce adverse perinatal outcomes or maternal inch-score and bile acids level in women with intrahepatic cholestasis of pregnancy [23]. After a secondary analysis of the PITCHES trial, which evaluated whether a particular group of women with ICP, based on their presenting characteristics, would benefit from treatment with UDCA, the report showed that there was no subgroup of women in whom the treatment had a beneficial effect on bile acid concentration or itch-score [23]. There could be other possible long-term benefits of using UDCA that are not yet fully understood. There are animal studies arguing that it may improve fetal dyslipidemia and lower the susceptibility of the adult offspring to metabolic disease later in life [24].

Rifampicin could be used as an alternative treatment, knowing that it improves symptoms and biochemical markers of liver injury in cholestatic liver disease outside pregnancy. It also enhances bile acid detoxification, an effect that is complementary to the upregulation of bile acid export induced by UDCA. There is an ongoing randomized study aiming to compare the effect of UDCA and rifampicin on maternal symptoms in severe early-onset ICP and to see if rifampicin can improve serum bile acids in association with UDCA [25].

Another new and challenging idea of therapeutic plasma exchange may lower potentially harmful bile acids and improve maternal itch secondary to the removal of pruritogens. However, the limited experience and potential complications, along with minimal sustained symptomatic benefit, could only support its future use for women with the most severe disease and no other treatment options [26].

Over-the-counter medications such as aqueous cream with menthol, lotions, or antihistamines such as chlorpheniramine, diphenhydramine, hydroxyzine, or promethazine, at night, are safe methods to relieve pruritus and they can even help with insomnia that typically accompanies severe pruritus.

Prothrombin time should be measured prior to delivery, especially with severe liver function tests modifications, particularly with jaundice or steatorrhea. Women with a prolonged prothrombin time should be advised to take $10 \mathrm{mg}$ of vitamin $\mathrm{K}$ orally, daily [10]. 
Management should be realized according to the peak of bile acid concentration, regardless of treatment with UDCA, when repeated bile acid testing is done in women with low risk of stillbirth [16]. All studies have considered peak total bile acids, so although the serum level gradually decreases naturally or due to treatment, this does not change the initial risk. Women with bile acids $<40 \mu \mathrm{mol} / \mathrm{l}$ can be reassured that they do not have an increased risk of preterm birth or stillbirth. Liver function tests, including bile acids, should be checked weekly, the risk should be reassessed at every follow-up and delivery should be planned at $37+0$ $-38+6$ weeks of gestation $[3,10,27]$.

In women with peak bile acids $>40 \mu \mathrm{mol} / \mathrm{I}$ there is an increased risk of preterm birth and neonatal unit admission and for women with peak bile acids $>100$ $\mu \mathrm{mol} / \mathrm{l}$ at any time during pregnancy, the risk of stillbirth is significantly higher than that of the general population [16]. The coexistence of pre-eclampsia or gestational diabetes can increase the risk of intrauterine death. The only intervention to affect adverse perinatal outcomes is likely delivery to be appropriately planned. Women with severe disease, but peak bile acid level < $100 \mu \mathrm{mol} / \mathrm{l}$, should be closely monitored and delivery should be recommended at 37+0 - 37+6 weeks of gestation, given that majority of unexplained fetal deaths occur after 37 weeks [28].

In women with total bile acid concentration during pregnancy $\geq 100 \mu \mathrm{mol} / \mathrm{l}$, most experts suggest delivery at $36+0-36+7$ weeks of gestation. However, delivery before 36 weeks should be considered, after extensive counseling, in women with excruciating pruritus not relieved after treatment, in case of deteriorating hepatic function (increasing transaminases or bile acids despite UDCA treatment) or in case of history of fetal demise before 36 weeks due to ICP [3]. Timing of delivery should be delayed as long as possible after 34 weeks of gestation. If delivery is recommended before 34 weeks, a course of antenatal corticosteroid therapy is indicated for reduction of neonatal respiratory morbidity and mortality. In contrast to pregnancies < 34 weeks of gestation, where consensus exists about antenatal corticotherapy, its use after 34 weeks is controversial given the absence of a survival benefit, less respiratory benefit due to the lower risk of serious respiratory problems and greater concern about potential long-term harm [3]. The American College of Obstetricians and Gynecologists (ACOG) recommends considering a single repeat course of corticosteroids in patients $<34$ weeks at imminent risk of preterm delivery within the next seven days with a prior course administered more than 14 days previously. If bile acids $>100 \mu \mathrm{mol} / \mathrm{l}$, but gestational age below 36 weeks, weekly monitoring of liver function tests should be performed until delivery.

Anticipating fetal demise remains the most difficult aspect in managing ICP. There is no evidence support- ing Doppler blood flow analysis in the uterine arteries, fetal umbilical, or cerebral arteries, not even in severe cases. No conclusive data is available about the effect of high serum bile acids on fetal heart rate variability, which is an established indicator of fetal autonomic nervous system function and wellbeing in utero [29], so cardiotocography is not relevant in this case, probably since the precipitating factor is an acute arrhythmogenic event and not a subacute or chronic disorder. Also, ultrasound screening for fetal growth or amniotic fluid volume does not predict fetal compromise nor does improve outcome. There is ongoing research regarding the use of fetal electrocardiogram (ECG) for monitoring the cardiac rhythm, but no official data has been published so far.

For all patients with ICP, serum bile acid levels and liver function tests should be performed 3-6 months postpartum. If bile acid levels remain elevated after 6 months, further evaluation is recommended to rule out an underlying genetic disorder or a chronic liver disease [8].

\section{CONCLUSIONS}

ICP is a relatively common disease during pregnancy with important implications for mother and child corelated to the severity of the disease. It has a complex etiology with strong evidence supporting genetic susceptibility. The most sensitive biomarker used for diagnosis are serum bile acids more than $10 \mu \mathrm{mol} / \mathrm{l}$.

Women should be categorized into different risk groups according to peak total bile acids and managed accordingly. Women with bile acids $<40 \mu \mathrm{mol} / \mathrm{l}$ can be reassured that they do not have an increased risk of preterm birth or stillbirth and they represent most cases. Women with severe disease (bile acids $>40 \mu \mathrm{mol} / \mathrm{I}$ but $<100 \mu \mathrm{mol} / \mathrm{I}$ ) should be closely monitored and delivery should be recommended at $37+0-37+6$ weeks of gestation. In women with total bile acid concentration during pregnancy $\geq 100 \mu \mathrm{mol} / \mathrm{l}$, most experts suggest delivery at $36+0-36+7$ weeks of gestation. However, delivery before 36 weeks, or even 34 weeks in very severe cases, should be considered after extensively conciliating the patient, in women with severe pruritus not relieved after treatment, in case of deteriorating hepatic function (increasing transaminases or bile acids despite UDCA treatment) or in case of history of fetal demise before 36 weeks due to ICP.

UCDA is the only approved treatment for ICP at this moment, although its benefits on fetal or maternal outcome are still debatable. Anticipating fetal demise remains the most difficult aspect in managing ICP. There is no evidence supporting Doppler blood flow analysis in the uterine arteries, fetal umbilical, or cerebral arteries, not even in severe cases. 


\section{REFERENCES}

1. Geenes V, Williamson C. Intrahepatic cholestasis of pregnancy. World $\mathrm{J}$ Gastroenterol. 2009 May 7;15(17):2049-66.

2. Sticova E, Jirsa M, Pawłowska J. New Insights in Genetic Cholestasis: From Molecular Mechanisms to Clinical Implications. Can J Gastroenterol Hepatol. 2018 Jul 26; 2018:2313675.

3. Lindor KD, Lee RH. Intrahepatic cholestasis of pregnancy. Available at: https://www. uptodate.com/contents/intrahepaticcholestasis-of-pregnancy?search= intrahepatic-cholestasis-\&source=search result\&selectedTitle=2 48\&usage_type= default \&display rank $=2$.

4. Jacquemin E, Cresteil D, Manouvrier S, Boute O, Hadchouel M. Heterozygous non-sense mutation of the MDR3 gene in familial intrahepatic cholestasis of pregnancy. Lancet. 1999 Jan 16;353(9148):210-1.

5. Nayagam JS, Williamson C, Joshi D, Thompson RJ. Review article: liver disease in adults with variants in the cholestasisrelated genes ABCB11, ABCB4 and ATP8B1. Aliment Pharmacol Ther. 2020 Dec; 52(11-12):1628-1639.

6. Bacq Y, Gendrot C, Perrotin F, Lefrou L, Chrétien S, Vie-Buret V, Brechot MC, Andres $\mathrm{CR}$. ABCB4 gene mutations and singlenucleotide polymorphisms in women with intrahepatic cholestasis of pregnancy. J Med Genet. 2009 Oct; 46(10):711-5.

7. Van Mil SW, Milona A, Dixon PH, Mullenbach $\mathrm{R}$, Geenes VL, et al. Functional variants of the central bile acid sensor FXR identified in intrahepatic cholestasis of pregnancy. Gastroenterology. 2007 Aug;133(2):507-16.

8. https://emedicine.medscape.com/ article/1562288-overview\#a5.

9. Williamson C, Geenes V. Intrahepatic cholestasis of pregnancy. Obstet Gynecol. $2014 \mathrm{Jul} ; 124(1): 120-133$.

10. Nelson-Piercy C. Handbook of Obstetric Medicine, 6th ed. CRC Press, 2020:220-224.

11. Falconer JD, Smith AN, Eastwood MA. The effects of bile acids on colonic motility in the rabbit. Q J Exp Physiol Cogn Med Sci. 1980 Apr;65(2):135-44.

12. Kirwan WO, Smith AN, Mitchell WD, Falconer JD, Eastwood MA. Bile acids and colonic motility in the rabbit and the human. Gut. 1975 Nov;16(11):894-902.

\section{Conflict of interest: none declared Financial support: none declared}

13. Glantz A, Marschall HU, Mattsson LA. Intrahepatic cholestasis of pregnancy: Relationships between bile acid levels and fetal complication rates. Hepatology. 2004 Aug;40(2):467-74.

14. Williamson C, Gorelik J, Eaton BM, Lab M, de Swiet M, Korchev Y. The bile acid taurocholate impairs rat cardiomyocyte function: a proposed mechanism for intra-uterine fetal death in obstetric cholestasis. Clin Sci (Lond). 2001 Apr;100(4):363-9.

15. Sepúlveda WH, González C, Cruz MA, Rudolph MI. Vasoconstrictive effect of bile acids on isolated human placental chorionic veins. Eur J Obstet Gynecol Reprod Biol. 1991 Dec 13;42(3):211-5.

16. Ovadia C, Seed PT, Sklavounos A, Geenes V, Di llio C, et al. Association of adverse perinatal outcomes of intrahepatic cholestasis of pregnancy with biochemical markers: results of aggregate and individual patient data meta-analyses. Lancet. 2019 Mar 2;393(10174):899-909.

17. Ropponen A, Sund R, Riikonen S, Ylikorkala $\mathrm{O}$, Aittomäki K. Intrahepatic cholestasis of pregnancy as an indicator of liver and biliary diseases: a population-based study. Hepatology. 2006 Apr;43(4):723-8.

18. Marschall HU, Wikström Shemer E, Ludvigsson JF, Stephansson O. Intrahepatic cholestasis of pregnancy and associated hepatobiliary disease: a population-based cohort study. Hepatology. 2013 Oct;58(4):1385-91.

19. Wikström Shemer EA, Stephansson O, Thuresson M, Thorsell M, Ludvigsson JF, Marschall HU. Intrahepatic cholestasis of pregnancy and cancer, immune-mediated and cardiovascular diseases: A populationbased cohort study. J Hepatol. 2015 Aug;63(2):456-61.

20. South Australia Maternal \& Neonatal Community of Practice. Obstetric Cholestasis. Available at: https://www. sahealth.sa.gov.au/wps/wcm/connect/ f91fbf004ee530b2a5ebadd150ce4f37/ obstetric+cholestasis_27042016.pdf?MOD= AJPERES\&amp;CACHEID=ROOTWORKSP ACE-f91fbf004ee530b2a5ebadd150ce4f37nxzrPx7.
21. Society for Maternal-Fetal Medicine Consult Series \#53: Intrahepatic cholestasis of pregnancy

22. Manna LB, Ovadia C, Lövgren-Sandblom A, Chambers J, et al. Enzymatic quantification of total serum bile acids as a monitoring strategy for women with intrahepatic cholestasis of pregnancy receiving ursodeoxycholic acid treatment: a cohort study. BJOG. 2019 Dec;126(13):1633-1640.

23. Fleminger J, Seed PT, Smith A, Juszczak E, et al. Ursodeoxycholic acid in intrahepatic cholestasis of pregnancy: a secondary analysis of the PITCHES trial. BJOG. 2021 May;128(6):1066-1075.

24. Borges Manna L, Papacleovoulou G, Flaviani F, Pataia V, Qadri A, et al. Ursodeoxycholic acid improves fetoplacental and offspring metabolic outcomes in hypercholanemic pregnancy. Sci Rep. 2020 Jun 25;10(1):10361.

25. Hague WM, Callaway L, Chambers J, Chappell L, Coat S, et al. A multi-centre, open label, randomised, parallel-group, superiority Trial to compare the efficacy of URsodeoxycholic acid with RIFampicin in the management of women with severe early onset Intrahepatic Cholestasis of pregnancy: the TURRIFIC randomised trial. BMC Pregnancy Childbirth. 2021 Jan 12;21(1):51.

26. Ovadia C, Lövgren-Sandblom A, Edwards LA, Langedijk J, et al. Therapeutic plasma exchange as a novel treatment for severe intrahepatic cholestasis of pregnancy: Case series and mechanism of action. J Clin Apher. 2018 Dec;33(6):638-644.

27. RCOG, Obstetric Cholestasis (Green-top Guideline No. 43/2011).

28. Kawakita T, Parikh LI, Ramsey PS, Huang CC, Zeymo A, Fernandez M, Smith S, Iqbal $\mathrm{SN}$. Predictors of adverse neonatal outcomes in intrahepatic cholestasis of pregnancy. Am J Obstet Gynecol. 2015 Oct;213(4):570.e1-8.

29. Vasavan T, Deepak S, Jayawardane IA, Lucchini M, et al. Fetal cardiac dysfunction in intrahepatic cholestasis of pregnancy is associated with elevated serum bile acid concentrations. J Hepatol. 2021 y;74(5):1087-1096. 